\title{
LA RACIONALIDAD LIMITADA EN EL USO DE LAS TARJETAS DE CRÉDITO EN EL ECUADOR
}

\section{LIMITED RATIONALITY IN THE USE OF CREDIT CARDS IN ECUADOR \\ Códigos JEL: G11 - G12}

\author{
Byron Villagómez Cevallos \\ Universidad Tecnológica Equinoccial \\ byrona.villagomez@ute.edu.ec
}

\begin{abstract}
RESUMEN
La teoría económica se basa en la existencia del denominado "agente representativo" que toma decisiones racionales. Sin embargo, se ha podido demostrar que muchas de las decisiones económicas que toman los consumidores se sustentan en el denominado "impulso", lo cual, les hace susceptibles de caer en el engaño y la manipulación, y en tal sentido la mencionada racionalidad del agente representativo, paradigma de la teoría neoclásica, pierde validez y es superada por la economía cognitiva. La metodología usada en esta investigación es en primer lugar bibliográfica y luego explicativa, basada en varias pruebas empíricas realizadas a varios grupos de estudio. Los resultados obtenidos fueron decisivos y guardan coherencia con estudios realizados en otros países (Akerlof, G., Shiller, R., 2015), esto es, que los consumidores que utilizan tarjetas de crédito se encuentran dispuestos a pagar más por los bienes y servicios que adquieren que aquellos que pagan en efectivo, que los beneficiarios de "avances de efectivo" no conocen las condiciones financieros de estos créditos de consumo y que los deudores morosos de cartera de consumo a través de los denominados avances de efectivo con tarjetas de crédito son acosados e intimidados por las entidades financieras.
\end{abstract}

\section{PALABRAS CLAVES:}

Agente Representativo, impulso, tarjeta de crédito.

\begin{abstract}
Economic theory is based on the existence of the so-called "representative agent" that makes rational decisions. However, it has been possible to demonstrate that many of the economic decisions made by consumers are based on the so-called "impulse", which makes them liable to fall into deception and manipulation, and in this sense the aforementioned rationality of the agent representative, paradigm of neoclassical theory, loses its validity and is overcome by cognitive economy. The methodology used in this investigation is first of all bibliographic and then explanatory, based on several empirical tests carried out in several study groups. The results obtained were decisive and are consistent with studies carried out in other countries (Akerlof, G., Shiller, R., 2015), that is, that consumers who use credit cards are willing to pay more for goods and services who acquire that those who pay in cash, that the beneficiaries of "cash advances" do not know the financial conditions of these consumer loans and that the delinquent debtors of the consumer portfolio through the so-called advances of cash with credit cards are harassed and intimidated by financial institutions.
\end{abstract}

KEY WORDS: Representative Agent, Impulse, Credit Card.

\section{INTRODUCCIÓN}


El presente artículo en primer lugar hace una crítica conceptual a una de las bases de la teoría económica neoclásica que parte de la existencia de un "agente representativo" maximizador de beneficios y poseedor de toda la información disponible para la toma de sus decisiones coherentes y racionales.

En contraposición, se habla más bien de la existencia de una racionalidad limitada y en tal sentido, el consumidor puede ser influenciado a tomar decisiones que afecten su bienestar y que perjudique sus intereses.

En consecuencia, en una economía de mercado, en donde los medios de comunicación tienen la posibilidad de ejercer una gran influencia en las decisiones de los consumidores a través de sus campañas de publicidad, deben existir mecanismos e instituciones que alerten al ciudadano y controlen de mejor manera el comportamiento empresarial.

En este caso, se habla del uso de las "Tarjetas de Crédito" y de los "Avances de Efectivo" y se presentan dos experimentos de la psicología social en donde se verifica que existe cierta manipulación deliberada, por parte de los emisores de dichos productos generando expectativas poco reales en los consumidores e indirectamente induciéndoles a tomar decisiones equivocadas de graves consecuencias para su bienestar.

Por otro lado, también se ha evidenciado cuando se realizaron varias entrevistas a clientes morosos con créditos de consumo reestructurado de tarjetas de crédito y/o avances de efectivo, que las entidades financieras no les exigen garantías que requieren trámites adicionales como certificados del registro de la propiedad o del registro mercantil ni garantes, ni siquiera la firma de una letra de cambio o pagaré, y en tales circunstancias cuando el cliente cae en morosidad, la entidad financiera tiene dos alternativas para la cobranza de sus recursos prestados, esto es, en primer lugar el acoso permanente por medios audiovisuales, teléfono, teléfono celular, e-mail, whats up, Messenger, instagram, todos los días incluidos feriados y varias veces al día y a diferentes horas, por meses e inclusive años, provocando en el cliente "moroso" una suerte de ansiedad y depresión clínicamente verificable; y en segundo lugar acudir a la instancia legal, que consiste en un juicio ordinario que puede demorar varios años.

Lógicamente la entidad financiera opta por la primera alternativa, la cual, inclusive en términos financieros de recuperación del dinero prestado, no resulta la mejor.

\section{METODOLOGÍA}

La presente investigación considera una metodología bibliográfica y explicativa.

Se pretende demostrar que uno de los supuestos básicos de la microeconomía, esto es, de la existencia del "agente representativo" racional y maximizador de beneficios, en el mundo real, no es correcto, ya que, gran parte de sus decisiones se hallan sustentadas en deseos, instintos, sentimientos y en la costumbre (heurística) y que por consiguiente la racionalidad de los agentes económicos es más bien limitada y necesitada de algún apoyo personal o institucional.

Luego, tomando como referencia los experimentos realizados por Akerlof, G y Shiller, a ciudadanos norteamericanos y revelados en su libro titulado "La Economía de la Manipulación" se hicieron varias pruebas a estudiantes universitarios ecuatorianos de la UTE, y encuestas a usuarios de tarjetas de crèdito, en donde se pudo constatar que los resultados obtenidos por los autores citados guardan coherencia con los aplicados a la realidad ecuatoriana.

Se debe expresar que para explicar el comportamiento humano, que muchas veces es contradictorio, no solamente es válido el uso de estudios econométricos (estilización de datos) que busquen relaciones de causalidad sino conforme lo establece la "Economía del 
Comportamiento" la psicología humana juega un papel fundamental y los experimentos con grupos de población representativos pueden dar más luces sobre su comportamiento.

En tal sentido, los experimentos efectuados que sustentan este artículo ofrecen una luz respecto a la necesidad de encontrar mecanismos de auto control y de regulación preventiva que posibiliten que los consumidores asuman sus derechos con mayor responsabilidad.

\section{MARCO TEÓRICO}

La teoría económica Neoclásica o tradicional que es aceptado por los agentes económicos parte de supuestos irreales, como la existencia del agente representativo maximizador de beneficios.

Todo lo resumen en un problema de maximización restringida, matematización de la ciencia económica.

Esta forma de ver la economía se remonta desde hace mucho tiempo, quizás con la idea de encontrar explicaciones en el comportamiento humano basadas en las ciencias exactas, de fácil comprobación.

Las teorías científicas difieren en 3 dimensiones principales:

a.- Ontológica,

b.- Epistemológica, y

c.- metodológica (Witt, 2008).

La dimensión ontológica hace referencia a los supuestos básicos sobre la estructura de la realidad. En economía, son supuestos sobre cómo son los individuos y sobre cómo interaccionan. Es ampliamente conocido que el supuesto de individuos idénticos (Agente Representativo), individualistas, egoístas y materialistas que interactúan en mercados perfecta e imperfectamente competitivos caracteriza la dimensión ontológica de la teoría neoclásica (Bunge. 2010).

Por otro lado, la dimensión epistemológica refleja la forma de organizar el pensamiento, es decir, cómo se definen los problemas para ser esquematizados y poder inducir hipótesis. En el caso de la teoría neoclásica, la dimensión epistemológica está constituida por el comportamiento optimizador.

Finalmente, la dimensión metodológica representa los métodos utilizados para expresar y verificar hipótesis. La teoría neoclásica emplea modelos matemáticos para expresar hipótesis y modelos econométricos para verificarlas.

En contraste con la teoría neoclásica, la teoría evolutiva neo - schumpeteriana (Nelson y Winter, 1982) difiere de aquella en sus dimensiones ontológicas y epistemológicas. El análisis evolutivo se centra en la teoría neo - schumpeteriana de la economía y no en otras teorías evolutivas, que también estudian el comportamiento económico, como son la teoría schumpeteriana (Schumpeter, 1912), las aproximaciones naturalistas (Veblen, 1898; GeorgescuRoegen, 1976, Hayek, 1971; North, 2005) o el darwinismo universal (Campbell, 1965).

Adicionalmente se considera el aporte realizado por Richard Talher (Premio Novel de Economía 2017), que considera que los agentes económicos para tomar decisiones óptimas podrían recurrir a los denominados "empujoncitos" (Nudge) regulatorios o de auto conocimiento y control.

\section{DISCUSIÓN}


La Teoría económica ortodoxa: se enfoca en la asignación eficiente de recursos, en un mundo de escasez y de infinito número de necesidades y su posterior afán por satisfacerlas como paradigma de la felicidad.

En el mundo real, los recursos no son escasos, sino mal distribuidos y por otro lado, la afirmación de necesidades ilimitadas, motiva a las empresas y a las agencias de publicidad a manipular al consumidor inundándole de información, la mayoría falsa, para inducirle a comprar, aun por encima de sus posibilidades.

En este punto aparece el mercado financiero, ofreciéndole al consumidor esa pastilla mágica llamada "Tarjeta de Crédito" y en particular el denominado "avance de efectivo"

La Tarjeta de Crédito ofrece un financiamiento de muy corto plazo (menor a 1 mes) y en cuanto a los "avances de efectivo" que en realidad son créditos de consumo, sus plazos no superan los 18 meses.

El consumidor incauto cuando cae en el juego de la "Tarjeta de Crédito" prioriza la satisfacción de sus gustos y preferencias actuales por sobre sus necesidades futuras.

El costo financiero de adquirir un bien o servicio a través de una tarjeta de crédito o recibir un "avance de efectivo" que en realidad es un crédito de muy corto plazo y en condiciones financieras prácticamente de usura, implica una serie de cargas financieras adicionales a la tasa de interés nominal, como son la "tasa de intercambio" que refleja el margen que recibe la entidad financiera por anticipar el dinero para la compra del bien o servicio y que es asumido por la entidad comercial en unos casos y generalmente por el propio consumidor, las comisiones por administración, tasas de mora, tasas de refinanciamiento cuando procede, etc.

Generalmente el consumidor que ha hecho uso de la tarjeta de crédito, al final del mes, no paga la totalidad de sus consumos sino que cae en el siguiente engaño del "PAGO MÍNIMO" y este es el principio del fin, ya que los valores no pagados en su totalidad sufren de un automático refinanciamiento a tasas de interés y comisiones nuevamente altas.

Luego si el cliente, requiere liquidez inmediata, puede caer en el tercer engaño, aceptar la oferta de "AVANCE DE EFECTIVO", sin reparar en las condiciones financieras a las que se verá sometido.

¿Cómo hizo el cliente para volver a caer en esta nueva manipulación? Pues muy fácil. No le dan tiempo a pensar, no le someten a un tiempo de espera mientras realizan una calificación de riesgo, no evalúan si el cliente está o no sobre endeudado antes de asumir esta nueva obligación, no requiere de garante, ni siquiera firma un pagaré o letra de cambio, y el dinero le entregan o le acreditan de inmediato. Maravilloso, verdad?? Hasta que llega el primer mes de pago, y resulta que el plazo del avance de efectivo en el mejor de los casos fue de 18 meses, la tasa de interés nominal anual del $20 \%$, intereses de mora $4 \%$, tasa de "intercambio" del $5 \%$ al $10 \%$ y un valor de la cuota impagable a no ser que se utilicen parte de los mismos recursos del anticipo solicitado para pagar las cuotas de esta nueva deuda.

Si el beneficiario de la tarjeta de crédito y sobre todo del "avance de efectivo" queda en mora, automáticamente entra a la "Central de Riesgos" y viene la persecución por todos los medios extra judiciales, llamadas por teléfono, directas o con grabaciones, una vez a la semana, para luego pasar a llamadas todos los días, atosigamiento por todos los medios electrónicos como email, whats up, Messenger, instagram, etc. y llegar al extremo de llamadas varias veces el mismo día desde muchas líneas de teléfono (no olvidar que el cliente se enfrenta a toda una infraestructura de cobranzas por parte de la entidad financiera o empresas tercerizadas) para sorprender al cliente moroso una y otra vez, incluidos feriados y fines de semana y esto por tiempo indefinido. 
Es importante indicar, que este acoso al que se ve sometido el cliente, es inconstitucional y que en otros países ha sucedido lo mismo y ha habido un cambio en la normativa respecto a la implementación de una prohibición de persecución e intimidación al cliente.

Es importante aclara que bajo ningún punto de vista se está afirmando que el crédito productivo bajo condiciones financieras aceptables es malo, todo lo contrario, la administración del crédito, bajo parámetros técnicos y de cooperación que busca el mutuo beneficio es positivo para la economía.

Lo que se afirma es que este tipo de crédito, basado en las tarjetas de crédito entregadas de manera indiscriminada a prácticamente toda la población ya sea que tenga bajos, medianos o altos ingresos y los denominados "avances de efectivo" si son nocivos para la sociedad en general, ya que prácticamente no tienen ningún control y se prestan para una gran manipulación de los medios de comunicación y de los intermediarios financieros que en su afán por maximizar sus beneficios son capaces de atentar contra la vida misma del consumidor.

La teoría económico ha avanzado bastante al respecto, y la economía del comportamiento claramente establece que los agentes económicos tienen una "racionalidad limitada" y que el tal sentido, sus decisiones, especialmente relacionadas con el consumo, no solamente son de responsabilidad del consumidor sino también de quien lo permite, que no le informa adecuadamente sobre los costos y beneficios de tales decisiones y lógicamente también del organismo de control que debería establecer mayores controles, normativas y programas extensivos de educación financiera.

Las pruebas experimentales realizadas en este artículo confirmaran la hipótesis de que los consumidores aceptan condiciones financieras perjudiciales a sus intereses y caen en el "impulso" y en el falso paradigma de vincular la felicidad con el consumo, exclusivamente.

\section{RESULTADOS}

1.- Una de las bases de la magia de la tarjeta de crédito es que la mayoría de nosotros piensa que compramos solo lo que necesitamos (o deseamos) y que no podríamos ser influidos por estímulos menores como por ejemplo si pagaremos con tarjeta de crédito o efectivo. Pero casi seguro estamos equivocados.

\section{¿Cómo sabemos que las tarjetas de crédito influyen en nuestro gasto de esta manera?}

Primero, existe la prueba circunstancial de que la gente con tarjetas de crédito gasta más. También se manifiesta que los portadores de tarjetas de crédito compraban más que los que pagaban en efectivo en sus visitas a los Centros Comerciales.

Pero estas diferencias en el gasto no pueden ser decisivas a la hora de responder a la cuestión de si las tarjetas de crédito hacen que la gente gaste más. Los portadores de tarjetas de crédito y los no portadores de tarjetas de crédito son diferentes y necesitamos saber que se trata de la tarjeta de crédito y no de esas diferencias, lo que causa el diferencial en pautas de gasto o de consumo.

Para resolver esta cuestión, se realizaron dos experimentos con estudiantes de una universidad privada; en el primero se los sometió a un estímulo subliminal vinculado a las tarjetas de crédito y se los comparó con la predisposición a gastar de un grupo de control sin estímulos sublimes. Para el estímulo se situó logos de MASTERCARD y VISA en la pantalla de un retro proyector en el aula en el que el grupo de estudio estaba trabajando. Se explicó que aquellos signos se 
estaban utilizando para otro experimento. Se preguntó después a los estudiantes cuánto gastarían en cuatro artículos representados en una imagen: un terno de hombre, una mochila, un short de caballero, unos zapatos deportivos. Cada artículo alcanzó una valoración mayor en el grupo de estudio que en el grupo no estimulado de control. (Tabla No. 1).

En un segundo experimento, se preguntó a los sujetos cuánto es lo máximo que estarían dispuestos a pagar (precio de reserva) por los mismos productos, se les enseñó los artículos en una pantalla y se midió el tiempo de sus respuestas.

Ante la presencia de una tarjeta de crédito en la pantalla del retroproyector, de nuevo los estudiantes estaban dispuestos a gastar más que el grupo de estudio no estimulado de control (Tabla No. 1)

TABLA No.1

\begin{tabular}{|c|c|c|c|c|c|c|c|c|}
\hline \multirow{2}{*}{\multicolumn{9}{|c|}{ GRUPO SIN ESTİMULO }} \\
\hline & & & & & & & & \\
\hline & MOCHILA & & \multicolumn{2}{|c|}{ ZAPATOS DEPORTIVOS } & \multicolumn{2}{|c|}{ PANTALONETA } & \multicolumn{2}{|c|}{ TERNO HOMBRE } \\
\hline & PRECIO & MAXIMO & PRECIO & MÁXIMO & PRECIO & MÀXIMO & PRECIO & MAXIMO \\
\hline PROMEDIO & 42 & 50 & 91 & 107 & 52 & 56 & 223 & 269 \\
\hline \multicolumn{9}{|c|}{ GRUPO CON ESTIMULO } \\
\hline PROMEDIO & 45 & 55 & 100 & 110 & 53 & 58 & 230 & 280 \\
\hline \multicolumn{3}{|c|}{ Fuente y Elaboración: Autor. } & & & & & & \\
\hline
\end{tabular}

Estas grandes diferencias en la predisposición a gastar explicaría por qué las tiendas aceptan felizmente las tarjetas de crédito, incluso si las empresas de tarjetas de crédito las gravan con una fracción significativa de la compra, con lo que se conoce como "tasa de intercambio".

En tercer lugar se realizaron encuestas a 10 ciudadanos que habían incurrido en el uso del "Avance de Efectivo" con una "Tarjeta de Crédito", obteniendo los siguientes resultados:

a.- El 100\% de encuestados si dispone de tarjeta de crédito, el 50\% American Express, el 40\% Mastercard y el $10 \%$ Visa.

b.- El 50\% de encuestados ha hecho uso del "Avance de Efectivo".

c.- El $60 \%$ ha destinado el uso del avance de efectivo para la compra de un bien o servicio (turismo), y el $40 \%$ restante para el pago de otras obligaciones de pago inmediato.

d.- El 90\% indicó que no se acordaba con exactitud el costo del financiamiento.

e- El 100\% no tuvo certeza en cuanto al plazo del último avance de efectivo que recibió.

f.- Ninguno de los encuestados conocía con exactitud el valor de la cuota que tenía que pagar por el "avance de efectivo",

g.- el $100 \%$ por ciento indicó que NO le pidieron ningún requisito adicional a más de tener la tarjeta de crédito para acceder al "Avance de Efectivo".

Como se puede apreciar de los resultados de estas encuestas, el cliente requiere de una liquidez inmediata sin interesarle las consecuencias de sus decisiones en el futuro cercano, que inclusive podría ser la fuente principal de las bancarrotas personales.

Como se ha visto, cada aspecto de esta industria financiera está implicada en la búsqueda de incautos. Empezando por los honorarios que cargan a los comerciantes, con sus cuantías enormes. Después van los consumidores que compran alegremente sus comestibles, calzado y cualquier otra cosa, con la tarjeta de crédito, extrayendo altas tasas de interés de aquellos con expectativas súper optimistas relativas a cómo pagarán la cuenta cuando llegue. Y después llueve sobre mojado con los cargos por mora y demás comisiones. En cada una de las etapas, la 
avidez competitiva por los beneficios explota nuestras debilidades y como colofón aparecen los "Avances de Efectivo".

Asimismo se realizaron varias entrevistas a deudores morosos de tarjetas de crédito ${ }^{1}, \mathrm{y}$ resultó elocuente la afirmación general de que a todos todavía no se les había iniciado ninguna acción legal a pesar de que en todos los casos ya habían pasado al menos 6 meses desde que dejaron de cancelar alguna cuota por dicha tarjeta de crédito. Lo que sí afirmaron todos, fue que han tenido una suerte de acoso permanente a través de los medios audiovisuales.

A nivel global, según el último reporte de la Superintendencia de Bancos, el Segmento de Crédito de consumo, durante el último año se ha incrementado en el 14\% (Tabla No. 2), con un valor promedio de usd. 20.200, 61; si se toma en cuenta que la tasa de morosidad de este tipo de consumo, el más alto de todos los segmentos, que es el que corresponde al de las Tarjetas de Crédito que es de alrededor del $5 \%$, se podría indicar que de esta cifra en promedio, se generarían USD. 1.010 de mora por cada operación crediticia.

\begin{tabular}{|c|c|c|c|}
\hline \multicolumn{4}{|c|}{ TABLA No. 2} \\
\hline \multicolumn{4}{|c|}{ MONTO PROMEDIO DE CRÉDITO } \\
\hline \multicolumn{4}{|c|}{ SEPTIEMBRE 2016 - SEPTIEMBRE DE 2017} \\
\hline TIPO DE CRÉDITO & sep-16 & sep-17 & D\% ANUAL \\
\hline COMERCIAL & $72.101,15$ & $73.084,92$ & $1 \%$ \\
\hline CONSUMO & $17.609,51$ & $20.200,61$ & $14 \%$ \\
\hline EDUCATIVO & $13.425,16$ & $11.601,63$ & $-15 \%$ \\
\hline INMOBILIARIO & $74.325,05$ & $75.626,94$ & $2 \%$ \\
\hline MICROEMPRESA & $2.861,81$ & $3.120,95$ & $9 \%$ \\
\hline PRODUCTIVO & $407.065,60$ & $575.497,91$ & $35 \%$ \\
\hline VIVIENDA INTERES PÜBLICO & $53.477,68$ & $56.497,91$ & $5 \%$ \\
\hline TOTAL & $640.865,96$ & $815.630,87$ & $24 \%$ \\
\hline \multicolumn{2}{|c|}{ Fuente: Superintendencia de Bancos } & & \\
\hline Elaboración: Autor & & & \\
\hline
\end{tabular}

En este mismo sentido, el informe de la Superintendencia de Bancos titulado: "Sistema de Bancos Privados, Reporte Comportamiento Crediticio Sectorial, Período: septiembre 2016 septiembre 2017, Análisis por Actividad Económica, páginas 8 y 9, textualmente expresan lo siguiente:

"En este periodo, el sistema de Bancos financió 1.531 Actividades, de las cuales la principal actividad atendida fue consumo (tarjeta de crédito y consumo no productivo) con un aporte del $34,76 \%$, este tipo de crédito no posee un desglose que permita conocer para que se destinan los fondos concedidos, seguido por Vivienda no productiva con el 9,05\% de participación en la cartera, Actividades de prestación de servicios con una participación del 30,97\%, Actividades de otorgamiento de crédito de instituciones dedicadas a la intermediación monetaria $(2,62 \%)$ y Venta de vehículos nuevos y usados con una participación del 2,49\%, las demás actividades económicas participan en la cartera de los bancos con un porcentaje menor al $2 \%$.

Las actividades económicas que presentaron la mayor contribución al indicador de morosidad además de Consumo fueron: Vivienda no productiva, Cultivo de palma africana, Educación no productiva, Actividades de prestación de servicios administrativos y Venta de vehículos nuevos y usados, con el $0,30 \%, 0,12 \%, 0,10 \%, 0,08 \%$ y $0,04 \%$, respectivamente."

\footnotetext{
${ }^{1}$ Una empresa dedicada a la cobranza de cartera de crédito bancaria accedió a facilitar información de algunos clientes de tarjetas de crédito que luego de muchas negociaciones habían llegado a firmar una acuerdo de pago, por la reserva del caso, en este artículo no se menciona ni a la empresa ni a los deudores.
} 
En definitiva es conocido que la cartera de consumo ha tenido un alto crecimiento durante los últimos años, en especial a través del uso de las Tarjetas de Crédito y justamente es este segmento el que presenta las más altas tasas de morosidad.

Obviamente las altas tasas de interés y los cortos plazos con que se determinan las condiciones financieras por usar estos productos provocan en gran parte dicho fenómeno.

\section{CONCLUSIONES}

1. La teoría económica neoclásica sustentada en la existencia del "agente representativo" y de que los consumidores disponen de toda la información relevantes y que toman decisiones racionales, es incorrecta, y justamente ésta equivocada forma de ver a los consumidores, ha servido para que las entidades financieras en su afán infinito de ganar emprendan en estrategias nocivas para los ciudadanos y les induzcan a tomar decisiones equivocadas.

2. La teoría económica del comportamiento más bien habla de que los agentes económicos disponen de una "racionalidad limitada" ya que sus decisiones en buena medida se ven influenciadas por el "impulso" y que en tal sentido, requieren del apoyo institucional para que sus decisiones les conduzcan a su beneficio.

3. Por otro lado, se ha observado que las entidades financieras otorgan el "avance de efectivo" que puede llegar hasta los USD. 10.000,00 sin un análisis de crédito estructurado y formal con cumplimiento de requisitos y documentos que pueden demorar varios días, como certificados de la propiedad, del registro mercantil, presencia de garante, firma de letra de cambio o pagaré, lo cual, aparentemente hace pensar que, a las entidades financieras no les interesa que el cliente piense y analice sino que tome una decisión basada únicamente en el "impulso".

4. La entidad financiera, cuando el cliente de este producto, cae en mora, al no tener las garantías necesarias, no puede iniciar un "juicio ejecutivo" que implica que el juez solamente revise la consistencia de las garantías y exija el pago inmediato caso contrario disponga las medidas cautelares del caso.

5. En consecuencia la entidad financiera, opta por el acoso y persecución al cliente moroso, que presumiblemente si quiere pagar pero no tiene la liquidez suficiente.

6. Aparentemente esta persecución no tiene consecuencias reales en el deudor moroso, que si quiere pagar pero que no puede; es peor, le provoca ansiedad e inclusive depresión.

7. Como último recurso y luego de varios años, la entidad financiera acude al juicio ordinario que demora varios años y que es de difícil pronóstico.

8. En tal sentido, inclusive a la entidad financiera, le conviene que previo al otorgamiento del "avance de efectivo" que en lenguaje simple es un "crédito de consumo" hacerle al cliente una "calificación de crédito" que obviamente demorará varios días y que por consiguiente podría ocasionar que el cliente con un poco más de análisis y consulta desista de estas operaciones de corto plazo y de alto costo financiero. 
9. Sería deseable que el organismo de control, llámese Superintendencia de bancos, tome cartas en el asunto y regule de mejor manera este producto financiero para beneficios no solamente de las entidades financieras sino y sobre todo de los clientes y de sus derechos ciudadanos.

\section{BIBLIOGRAFÍA}

1.- Arrow, K., 1962. Economic Welfare and the Allocation of Resources for Invention, NBER, Washington.

2.- Witt, U., 2008. What is specific about evolutionary economics? Journal of Evolutionary Economics, Springer, XVIII, 547-575.

3.- March, J.G., Simon, H. A., 1958, Organizations, Wiley, New York.

4.- Nelson, R. R., 1991. Why do firms differ and how does it matter? Strategic Management Journal, XXII, $61-74$.

5.- Nelson, R.R., 1993. National Innovation System. Oxford University Press, New York.

6.- Nelson, R. R:, Winter, S. G., 1982. An Evolutionary Theory of Economic Change, Harvard University Press, Cambridge, MA.

7.- Schumpeter, J.A., 1912. Theory of Economic Development, Harward Univ. Press, Cambridge, MA.

8.- Freeman, C., Soete, L., 1997. The Economics of Industrial Innovation, $3^{\mathrm{a}}$ Edit. Pinter, Londres.

9.- Fernández, J. 2015, Economía neo-schumpeteriana, innovación y política tecnológica. Cuadernos de Economía. ELSEBIER.

10.- Akerlof, G., Shiller, R., 2015, La Economía de la Manipulación, Editorial Planeta, Bogotá.

11http://oidprd.sbs.gob.ec/medios/PORTALDOCS/downloads/articulos_financieros/Estudios\% 20Sectoriales/2017/ES_BP_sept_2017.pdf 